\title{
Analytical approaches to ensure product quality - AAPS Joint Face-to-Face Meeting of the Stability, the Pharmaceutical Impurities, and the CMC Statistics Focus Groups, April 6 ${ }^{\text {th }}, 2016$ in Gaithersburg, MD
}

Kim Huynh-Ba*, Greg Larner, Helen Strickland, Dilip Choudhury, Yan Wu, Jeff Hofer, Zhenyu Wang and Timothy Schofield

\begin{abstract}
An AAPS joint face-to-face meeting was held on April 6th, 2016 at Medlmmune, Gaithersburg, MD. The meeting was organized by members of the Steering Committees of the Stability Focus Group, the Pharmaceutical Impurities Focus Group and the Chemistry, Manufacturing and Controls (CMC) Statistics Focus Group. Twenty two subject-matterexperts (SME) from the Industry and the Food \& Drug Administration (FDA) participated as discussion facilitators or discussion leaders. The total participants reached 65 people representing various sizes of pharmaceutical companies. Four contemporary technical topics on analytical approaches to ensure product quality were selected to be discussed and shared information: (1) Approaches to set release limits and managing method variability; (2) Ensure product quality through lifecycle management; (3) Best practices for predictive stability programs; and (4) ICH Guideline M7 and mutagenic impurities.

The purpose of the meeting was to share the best practices in the industry related to the topics; therefore, it is not intended for the participants to reach a consensus on the key issues discussed. This paper summarizes the discussions in the meeting.
\end{abstract}

\section{Background}

An AAPS joint face-to-face meeting was held on April $6^{\text {th }}$, 2016 at MedImmune, Gaithersburg, MD. The meeting was organized by members of the Steering Committees of the Stability Focus Group, the Pharmaceutical Impurities Focus Group and the Chemistry, Manufacturing and Controls (CMC) Statistics Focus Group. Four contemporary technical topics were selected to be discussed. Twenty two subject-matter-experts (SME) from the Industry and the Food \& Drug Administration (FDA) participated as discussion facilitators or discussion leaders (AAPS Joint Face-to-Face meeting summary, 6-April-2016, part I; AAPS Joint Face-to-Face meeting summary, 6-April-2016, part II).

\footnotetext{
* Correspondence: kim.huynhba@pharmalytik.com
}

Pharmalytik LLC, Newark, DE, USA
Topic A: Approaches to set release limits and managing method variability towards ensuring product quality ICH Q1E provides regulatory guidance for calculating shelf life of a drug product or retest period of a drug substance based on stability data utilizing simple statistical approaches. To ensure that the drug products manufactured over the life cycle consistently meet the registered specification during its shelf life, it is important to have an internal control limit for the assay that factors in typical manufacturing and analytical variabilities. This is typically called "release limit" which is a range of potency within which an individual lot can be released to market ensuring that its potency will remain within registered specification limits throughout the shelf life. Thus "release limit" provides an internal control for ensuring product quality (Fig. 1). The impurity level increases over the shelf life as a combination of the loss of active and the uncertainty of 


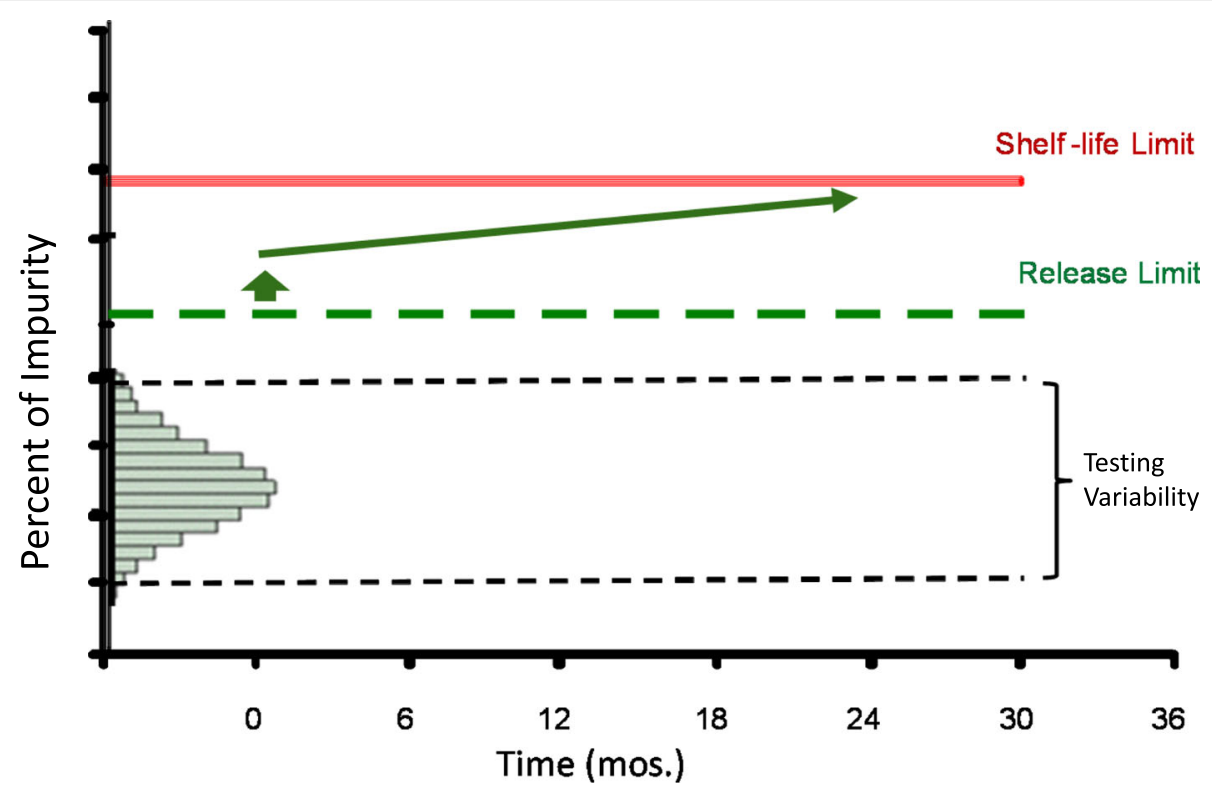

Fig. 1 Release Limit as an internal control

release assay procedure. A number of statistical approaches have been described for calculating release limits, a commonly used one is Allen, Dukes and Gerger method (Allen et al. 1991). There is an inconsistency in establishing harmonized control strategies for release limit from organizations working globally.

The participants agreed that development of a release limit is critical for controlling the product quality; however, the data utilized and procedure used to determine an appropriate release limit is not consistent. This is not unexpected since release limit is an internal control parameter. The following points were raised and discussed by the participants.

1. The stability profile of the drug product shows change over time, the typical variabilities in stability profile are incorporated in the calculation of shelf life by ICH Q1E. The regression/covariance approach described in ICH Q1E is one way of characterizing the stability change over time and its associated variability. The shelf life is a key factor in the calculation of release limit. Manufacturing data can be used to assess the capability of meeting the calculated limit based (based on the distribution/ histogram of Fig. 1), but it is not a component of the calculation. One must work backwards-given a shelf life, changes in stability profile over time, specification range for assay and impurities, manufacturing variability and the uncertainty in calculation of a release limit following the Allen, Duke, Gerger approach (Allen et al. 1991).
2. The drug substance stability profile is important. The maximum allowed level of impurities in the drug substance is an important consideration. Typically the drug substance attribute (assay) is used in formulating the batch during manufacture.

3. Analytical variability is an important consideration. The typical analytical variability as established during method validation should be considered in calculating the release limit.

The participants agreed that having an appropriate release limit would ensure consistent quality of the drug product through the shelf life and thus protect the manufacturer and the patient. It is important to have batch data from significant number of commercial batches using the final process in calculating the release limit. When a release value falls outside of a release limit, the quality system will prompt an investigation and mitigation procedure will be developed.

In summary, the majority of the companies used certain released limits; of those, approximately 50\% uses statistical approaches to establish release limits, others use a variety of alternative methods; however, specific non-statistical approaches were not discussed. However, it was concurred that we need to establish a common understanding of terminology.

Topic B: Strategies for ensuring global product quality through effective life cycle management The Guidance for Industry entitled "Analytical Procedures and Methods Validation for Drugs and Biologics" 
was published by FDA on 27 July 2015 (Food and Drug Adminstration, Guidance for Industry 2015). Participants discussed the key challenges experienced in product lifecycle management and best practices. They shared typical experiences with analytical methods lifecycle supporting product lifecycle management and explored best approaches to global product lifecycle management.

1. Several large pharma companies have developed systematic analytical quality-by-design (AQbD) approach for small and large molecule areas. This approach adds significant value to method development by defining and understanding critical method attributes, critical method parameters, and method operable design region. Based on the experiences by several companies, it was recognized that regulatory flexibility cannot be realized at this time from AQbD based method development and validation consistent with ICH Q8, 9 and 10 . (International Conference on Harmonisation of Technical Requirements for Registration of Pharmaceuticals for Human Use \& Q8 (R2) 2009; International Conference on Harmonisation of Technical Requirements for Registration of Pharmaceuticals for Human Use \& Q9 2005; International Conference on Harmonisation of Technical Requirements for Registration of Pharmaceuticals for Human Use \& Q10 2008) Best practices were discussed on clinical phaseappropriate method validation approaches to support product development as follows:

- Phase I: Simplified documentation practices, for example, scientific method qualification plans are used in place of formal protocols; platform standard method conditions are used demonstrating that the methods are fit-for-purpose at this phase.

- Phase II: general protocols issued by analytical development groups, pre-approved template protocols are used by several companies, demonstrate that the methods are fit-for-purpose.

- Phase III: formal protocols, QA approved; fully validated methods

2. Science and risk-based approach should be used to drive better decision making for current product development process. It is important to balance the needs of method changes, technology/process improvement, and manufacturing site changes, etc. with product understanding and regulatory expectations to ensure consistent product quality throughout the lifecycle. The following issues were discussed: 2.1 Possibility of obtaining regulatory flexibility from AQbD-based method development was discussed; however, it was recognized that regulatory flexibility is not typically granted by most agencies at this time.

2.2 FDA often observes additional development activities after approval; and request for introduction of the changes in analytical methods and process are made. It was recognized that introduction of newer analytical technologies may be beneficial to ensure consistency of product quality throughout the product lifecycle. However, it was agreed that when analytical methods based on newer technologies (e.g., UPLC, SFC etc.) are proposed for commercial products, one needs to consider the lead time and build a business case (time saving, training needs, implementation feasibility etc.) for introducing the technology. Commercial sites are heavily invested in established technologies both in capital equipment and staff training; therefore planning to implement a new technology into an established, multi-product, facility may take 2 years or more of lead time.

2.3 Predictive or accelerated stability studies may be considered for annual stability batches to help monitor process control and identify potential issues early. Several participants also discussed success in gaining regulatory approval for "Lean stability approach" (e.g., stability protocol limited to monitoring parameters that changes on storage) as post approval changes with adequate data including process control and scientific justification. Regulatory SME's concurred that with sufficient data and clear justification, stability protocols for post-approval annual stability batches can be "leaner" and have "non-value-added" tests eliminated.

2.4 For biologic development, it was noted that accelerated (unfrozen) API stability studies as compared to frozen API are very informative for identifying potential changes to be anticipated; however, it is not necessary for formal stability protocol. Such studies are also useful for understanding potential impact of short temperature excursions resulting from handling of the API for normal use over the life of a batch. Frozen API is inherently stable, thus long term stability study does not add much scientific value. (Kochling et al. 2016)

3. Opportunities and challenges of meeting evolving regulatory requirements and compliance standards in a global environment

3.1 Life cycle management of a global product often requires managing multiple specifications required for different countries (International Conference on Harmonisation of Technical Requirements for Registration of Pharmaceuticals for Human Use, Q12 Concept Paper 2014). Best practices to 
manage multi-specification products used by different companies were discussed. It was concurred that companies need to think both global (for general strategy) and local (for meeting specific local country-specific requirements) simultaneously. Pfizer has coined the term "Glocal" to encourage such thinking. To manage multiple specifications, some companies maintain one specification that meet or exceed specification requirements of each country, others manage individual country specifications. Multispecification approach adds significant complication to managing commercial supply chain.

3.2 When multiple specifications are needed for products in different markets, companies typically test to the tightest specification. With this practice, one set of release test data meets all global specification requirements. In addition, with this practice, the innovators may hold their products to a tighter specifications compared to generics, which may be approved for a specific market that allows less rigorous specifications. It is noted that while regulatory agencies observe and review each other's approval decisions, FDA makes their own decision independent of other agencies' decisions.

Overall, the attendees concurred that lifecycle management approaches for global products present significant challenges and there are differences in the best practices among companies. It was agreed that further discussions on this subject in future forums will be very beneficial.

\section{Topic C: Challenges and Best Practices for Successful Predictive Stability Programs (small molecules and large molecules)}

Predictive stability has long been used to quickly generate information to make timely and science-based decisions. At each breakout session, a case study of implementation was presented to highlight a successful approach to the topic and illustrate how these data can be leveraged for better decision making and/or time and resource savings. For example, when there is no physical changes in the dosage form occur, then the Arrhenius model for chemical reaction can be used. However, when physical changes occur, the reaction rates typically do not follow Arrhenius model, then a model can be designed to predict stability profile throughout the expiration dating period. With such model, predictive stability studies can support the selection of packages and/or formulations, to justify excursions and provide insight on in-use stability (Genton \& Kesselring 1977; Waterman et al. 2007). In addition, these data could also be used to justify the retest period for clinical trial material and to justify lean post-approval protocols.

Predictive stability data can also be used to help in the establishment of degradation mechanism, identification of the degradation pathways, development of stability indicating method, and to support accelerated development programs. Development of these predictive models is very beneficial to gain a better understanding of how the product behaves on long term stability. However, the implementation of predictive stability study faces challenges. Table 1 list examples of challenges that have been encountered. For example, one is working with a stable product, thus changes on stability is not significant. Therefore, it would be difficult to develop an accurate predictive model as these minute changes can be concealed by noise. There is also a fear that an unsuccessful model can cast doubt on activities of other products.

Different approaches are recommended to overcome resistance of using predictive models. One is to conduct an initial study to determine critical activities. Risks can also be reduced by running additional tests outside of formal stability program and providing data or setting up testing if challenged by a regulatory agency. Participants also discussed unique issues pertaining to large versus small molecules development. Table 2 lists the typical issues presented. For example, Arrhenius model is not applicable for large molecules or the variability of quantitate methods for biologics is too large and make it difficult to narrow down to a precise predictive model. Other challenges would be non-linear model of which extrapolation from accelerated condition to label storage condition becomes risky.

Flexibility in data reporting was also discussed as it can be key to enable successful predictive stability programs. It is noted that reporting below Limit of Quantitation (LOQ) can provide several advantages. Extra decimal places can also be helpful; however, this can be a barrier as there may be challenges with existing LIMS

Table 1 Example of challenges encountered using predictive stability studies

- Mistrust of models-lack of understanding-or too complex to model
- Lack of broad champion
- Lack of resources or different use of resources
- Lack of knowledge on approach
- Risk aversion
- Data quality is questioned if using low levels
- Product is too stable-difficult to develop predictive degradant
model when change is small relative to noise
- If modeling is not successful for a product it may cast doubt on
other products
- Challenges modeling dissolution


Table 2 Unique issues for large versus small molecules

- Applicability of Arrhenius equation for large molecules

- Perceived need to start with first principles

- Variability for biologics may make it more difficult to precisely model

- Fundamental model selection for biologics

- Lack of trust of non-linear models

- Temperature and/or Humidity is too high thus modeling is not predictive.

- Physical changes/polymorph changes-modeling in solids

- Modeling is difficult for proteins in solution-reversible step vs irreversible steps

- Label condition for Biologic drug substances is $-20 \mathrm{C}->$ what is accelerated?

- Extrapolation can be risky

default processing or reporting data above Limit of Detection but below LOQ.

Best practices were recommended to use multiple preparations rather than multiple injections when replication was desired to reduce variability and improve estimation. When possible, it was recommended to hold samples and test together on one run to remove day to day variability. It was also noted that variability of biologic testing may make it difficult to develop a precise predictive model.

Overall, all agreed that significant opportunities exist to leverage predictive stability; however, one should start with a small program and build upon successes to grow the program. This topic seems to gain much interest from participants and warrants future discussion for industry and regulatory perspectives.

\section{Topic D: ICH M7 and Mutagenic Impurities}

This group started by presenting the scope of the $\mathrm{ICH}$ M7 guideline (International Conference on Harmonisation of Technical Requirements for Registration of Pharmaceuticals for Human Use, M7 (R1) 2015). It applies to new drug substance and drug product during clinical development and subsequent applications for marketing, as well as specific cases of post-approval submissions of marketed products and to new marketing applications. Figure 2 shows the general framework of the guideline (Miller et al. 2015).

This guideline provides assessment on potential impurities such as starting materials, reagents and intermediates downstream of the starting material, in addition to other identified impurities. Actual impurities are those that have been identified as per levels stated in $\mathrm{ICH}$ Q3A/B.

Both potential and actual impurities should undergo a hazard assessment to determine their classification (-6) with respect to mutagenic and carcinogenic potential and then undergo a risk characterization to set appropriate limits. For Class 1, 2 and 3 impurities, ICH M7 guideline provided the principles of risk characterization that are used to derive acceptable intakes (Table 3).

Four options to control potential impurities are discussed.

1. Monitor the impurity in the drug substance with acceptance criteria $\leq$ acceptable limit

2. Monitor the impurity in the intermediate, starting material or in-process control with acceptance criteria $\leq$ acceptable limit

3. Include impurity on a starting material, intermediate or in-process control specification with an acceptance criteria above the acceptable limit coupled with well understood purge.

4. No testing, control assured via chemistry and process understanding

This group concluded by discussing and clarifying the scope of the guidance, i.e. excipients, impurities in biologics, if the impurity is a small molecule. It was noted that communication across disciplines (e.g.

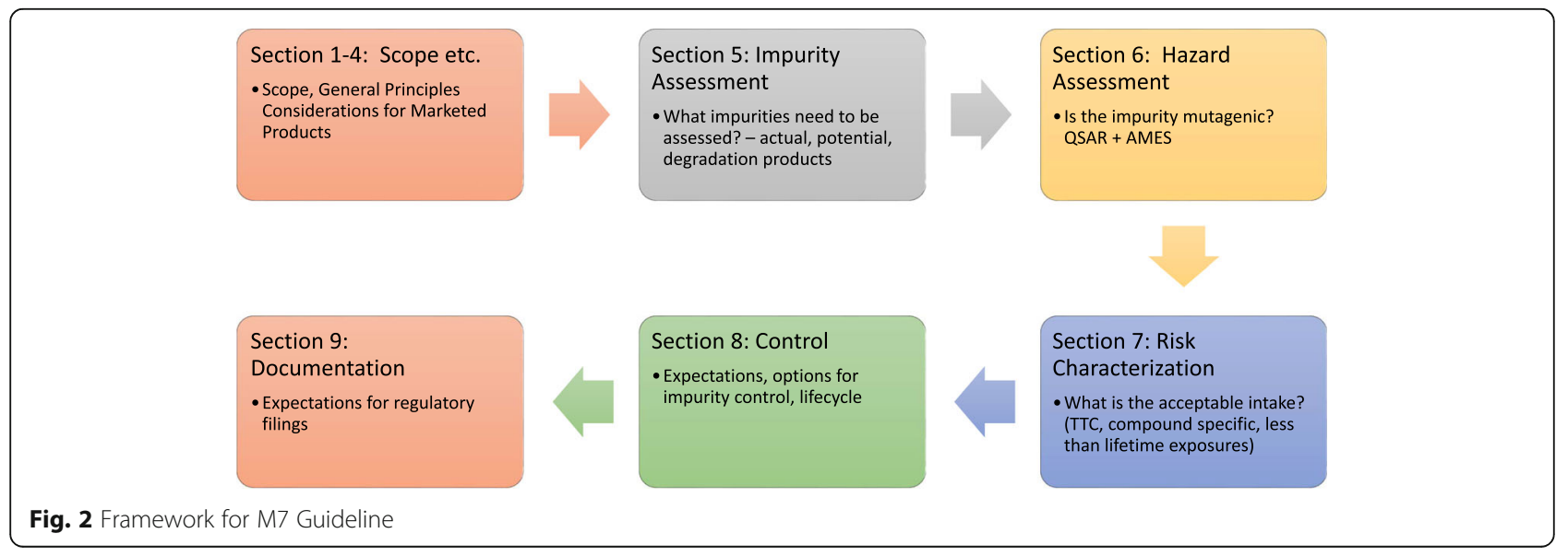


Table 3 Risk characterization for acceptable intake levels

- TTC-based acceptable intakes
- Develop compound-specific limits when carcinogenicity data available
- Acceptable intakes based on compound-specific risk assessments
- Acceptable intakes based on Less than lifetime (staged TTC) limit concept
- Exceptable total daily intakes for multiple impurities

clinical, $\mathrm{CMC}$, toxicology) is key to ensure appropriate controls are in place, especially since dose levels may change or the drug may be used in combination with others or for a different indication. Challenges with analytical method, e.g. method transfer, capabilities of QC lab, were noted. There was much discussion around when to identify an impurity and when to perform a toxicology assessment using Quantitative Structure-Activity Relationship ((Q)SAR) method, e.g. whether to wait until the impurity is confirmed or decide it should be routinely done at certain phase of development. Inclusion of information in the filing, i.e. stage dependence, placement, materials/reagents prior to route disclosed also discussed.

Discussion also included the use of (Q)SAR software. Participants shared that FDA recommended to report the model name, version, description, methodology and outcome. Additional scientific justification needed if Sponsor is "over-ruling" the result by (Q)SAR method. It is noted that FDA uses the most recent (Q)SAR software version; therefore, as feasible, Sponsors may consider rerunning predictions for impurities above threshold when a new software version is released. Although ICH M7 clearly states when assessment needs to be done on a marketed product, Sponsors have seen some queries particularly from EMA requesting assessment even when not warranted as per the guidance.

Suggestion was made for the Pharmaceutical Impurities Focus Group to consider a technical session to discuss real world problems in implementing ICH M7 such as best demonstrated practices, Agency recommendations, and case studies to continue this discussion.

\section{Overall conclusion}

The meeting was concluded on a high note. Participants appreciated the opportunity to have face-to-face interactions with multiple subject-matter-experts from the industry and FDA representatives. Technical information and common practices are shared and received positively at all breakout sessions. It is not expected for participants to reach a consensus due to the time limitations. The three sponsored AAPS Focus Groups have developed several plans to continue the discussion of these critical topics at upcoming AAPS annual meeting and workshop.

\section{Acknowledgements}

The authors gratefully acknowledge the support from all the Subject Matter Experts that participated in the Face-to-Face discussion. We also wish to thank Timothy Schofield (GSK) and Fadi Hakki (Medlmmune) for organizing the venue, Elizabeth Scuderi (AAPS) and Jackson Harar (AAPS) for managing the registration, and Anita Ymbert (Medlmmune) for on-site logistic support.

\section{Authors' contributions}

$\mathrm{KH}-\mathrm{B}$ and $\mathrm{DC}$ were the primary authors of this meeting report. All authors read, commented and approved the final manuscript.

\section{Competing interests}

All authors declare that they are employees or owners of the companies/ institutions provided and therefore received payments from said companies/ institutions. Otherwise, the authors declare that they have no competing interests.

Received: 26 August 2016 Accepted: 2 January 2017

Published online: 14 January 2017

\section{References}

AAPS Joint Face-to-Face meeting summary, 6-April-2016, part l, http://www.aaps. org/uploadedFiles/Functional/AAPS_Focus_Group_Discussion_Board/Stability_ Focus_Group/2016\%20Final\%20F2F\%20Joint\%20Meeting\%20Highlights_final_ \%20part\%201.pdf. Accessed 8 Jan 2017

AAPS Joint Face-to-Face meeting summary, 6-April-2016, part II, http://www.aaps. org/uploadedFiles/Functional/AAPS_Focus_Group_Discussion_Board/Stability_ Focus_Group/2016\%20Final\%20F2F\%20Joint\%20Meeting\%20Highlights_final_ part\%202.pdf. Accessed 8 Jan 2017

Allen PV, Dukes GR, Gerger ME (1991) Determination of Release Limits: A General Methodology. Pharm Res 8(9):1210-1213

Food and Drug Adminstration, Guidance for Industry (2015) Analytical Procedures and Methods Validation for Drugs and Biologics

Genton D, Kesselring UW (1977) Effect of Temperature and Relative Humidity on Nitrazepam Stability in Solid State". J Pharm Sci 66(5):676-680

International Conference on Harmonisation of Technical Requirements for Registration of Pharmaceuticals for Human Use, Q10, "Pharmaceutical Quality System", Step 4, June 2008. http://www.ich.org/fileadmin/Public_Web_Site/ ICH_Products/Guidelines/Quality/Q10/Step4/Q10_Guideline.pdf

International Conference on Harmonisation of Technical Requirements for Registration of Pharmaceuticals for Human Use, Q12 Concept Paper (2014) Technical and Regulatory Considerations for Pharmaceutical Product Lifecycle management

International Conference on Harmonisation of Technical Requirements for Registration of Pharmaceuticals for Human Use, Q8 (R2), "Pharmaceutical Development", Step 4, August 2009. http://www.ich.org/fileadmin/Public_Web_ Site/ICH_Products/Guidelines/Quality/Q8_R1/Step4/Q8_R2_Guideline.pdf International Conference on Harmonisation of Technical Requirements for Registration of Pharmaceuticals for Human Use, Q9, "Quality Risk Management", Step 4, November 2005. http://www.ich.org/fileadmin/Public_ Web_Site/ICH_Products/Guidelines/Quality/Q9/Step4/Q9_Guideline.pdf

International Conference on Harmonisation of Technical Requirements for Registration of Pharmaceuticals for Human Use, M7 (R1), "Assessment and Control of DNA Reactive (mutagenic) Impurities in Pharmaceuticals to Limit Potential Carcinogenic Risk", step 4, June 2015

Kochling J et al (2016) A platform analytical quality by design (AQbD) approach for multiple UHPLC-UV and UHPLC-MS methods development for protein analysis". J Pharm Biomed Anal 125:130-139

Miller S (2015) Food and Drug Administration, Assessment and Control of Mutagenic Impurities in Pharmaceutical Products - Implementation of ICH M7 Guideline, 2015 AAPS Annual Meeting, Orlando

Waterman KC et al (2007) Improved Protocol and Data Analysis for Accelerated Shelf-Life Estimation of Solid Dosage Forms. Pharm Res 24(4):780-790 